\title{
About the characterization of urban public transport networks and their terminals
}

\author{
Ş. Raicu, V. Dragu, Ş. Burciu \& C. Ştefănică \\ Department of Transport, Traffic and Logistics, \\ University "Politehnica" of Bucharest, Romania
}

\begin{abstract}
The underground network, like a high capacity infrastructure network, which is part of the public urban transport networks, plays a major role in any strategy of increasing public transportation attractiveness. The upcoming development of the Bucharest underground network stimulated the present research. We tried to emphasize the attractiveness of the designed network in relation to the existing one. In a comparative approach, on a system/network level, the essential properties of the network have been studied: ubiquity, connexity, connectivity, homogeneity/isotropy, vulnerability and the different rank of the nodes (generalized accessibility, Shimbel accessibility and nodal accessibility). Mostly because of the way ubiquity, vulnerability and isotropy studies have been conducted, original theoretical aspects could be revealed. The study's conclusions are useful to fundament network development solutions and also to increase collaborative aspects of the services offered to passengers and to establish priorities in maintenance activity.
\end{abstract}

Keywords: underground network, ubiquity, vulnerability, accessibility, isotropy.

\section{Introduction}

Designing and realizing transport infrastructure networks, like any other technical infrastructure of society, must obey a land use principle according to the social-economical needs of the society. The network, no matter the flows residing on it, must be considered a consequence of a land use planning requirement that establishes a connection between technical capabilities and territory service $[5,9,11]$. 
The Bucharest underground network, which recently celebrated the $30^{\text {th }}$ anniversary of the first line $\left(16^{\text {th }}\right.$ November 1979), has a particular history. The project and the construction itself (including gears and transport vehicles) carry the landmark of the isolation of our country in that period. That is why decisions regarding the structure and the priorities in achieving the lines were almost exclusively technical. Correlation with major passenger flows was not a determinant argument for the underground network configuration (fig.1). Even the link with the central railway station was not a priority, being realized later, as well as the flows with origins in high-density residential areas or the links with Aurel Vlaicu or Henri Coanda airports not being priorities for the underground network development [10].

The lack of correlation of people's major mobility needs is obvious even in the way stations have been located (distances between them of almost $1.5 \mathrm{~km}$, reducing attraction). Although other stations were later built, the lack of correlation between technical achievement and people's need for mobility still persists.

The growth of the new residential areas and commercial centres intensified the discrepancies between mobility demand and underground network offer. The highly congested road network of Bucharest and the metropolitan area imposed underground network development. So, by 2030, it would have the configuration from figure 2 , and the synthetic characteristics from table 1 .

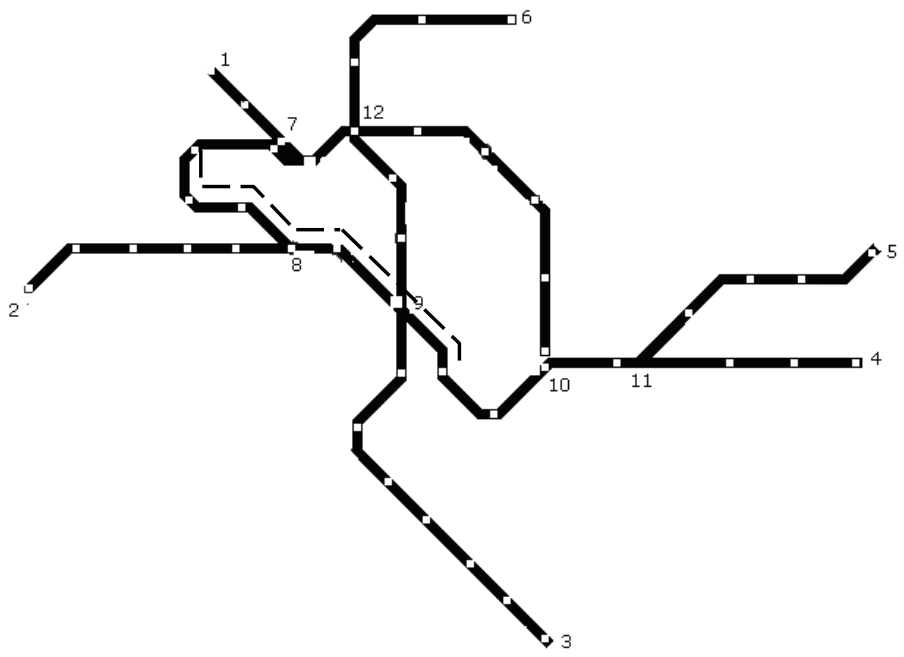

Figure 1: Underground network (present situation). 
Table 1: $\quad$ Underground network characteristics.

\begin{tabular}{|c|c|c|c|c|c|c|}
\hline Network & $\begin{array}{c}\text { Length } \\
{[\mathbf{k m}]}\end{array}$ & $\begin{array}{c}\text { No. of } \\
\text { nodes }\end{array}$ & $\begin{array}{c}\text { No. of } \\
\text { links }\end{array}$ & $\begin{array}{c}\text { No. of } \\
\text { stations }\end{array}$ & $\begin{array}{c}\text { Density } \\
{\left[\mathbf{k m} / \mathbf{k m}^{\mathbf{2}}\right]}\end{array}$ & $\begin{array}{c}\text { No. of } \\
\text { lines }\end{array}$ \\
\hline Existing & 66,95 & 12 & 13 & 55 & 0,29 & 4 \\
\hline Projected & 106,75 & 24 & 30 & 103 & 0,46 & 6 \\
\hline
\end{tabular}

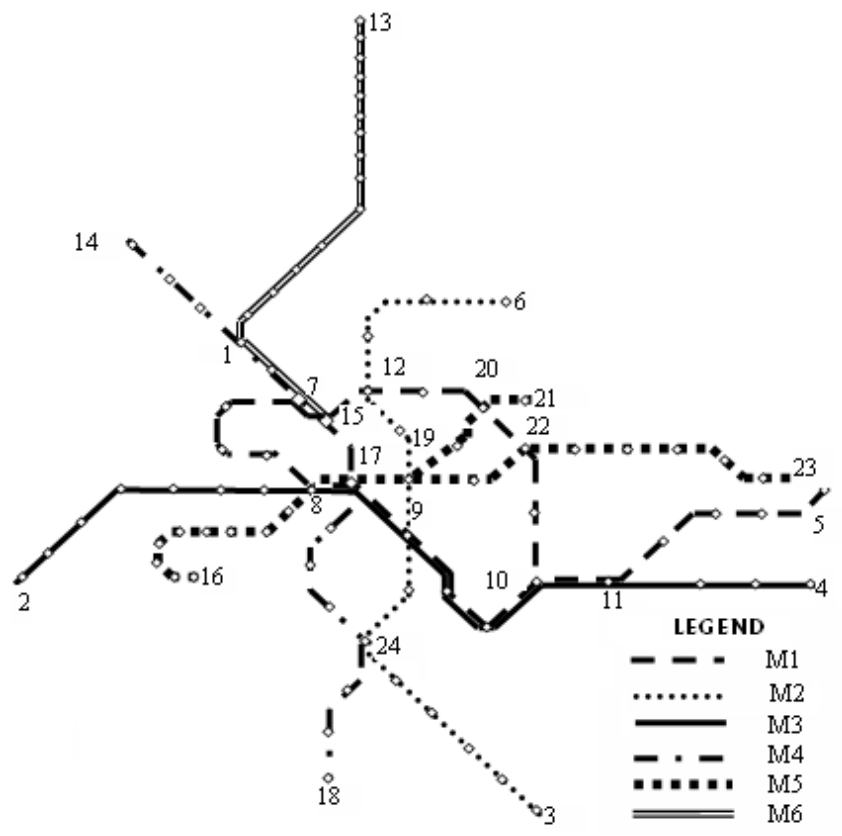

Figure 2: $\quad$ Underground network (with 2030 extensions).

\section{Properties of the networks}

\subsection{Ubiquity}

The ubiquity is the network's property of being accessible in more points in the same time/for a certain period.

The degree of ubiquity reflects the spatial covering of the network. Underground network ubiquity, like for the others networks of terrestrial transportation, is strictly determined by stations' locations.

In the simplest approach, that of a continuous space, it is obvious that increasing the degree of ubiquity for the network by increasing stations density (by decreasing the distance between the stations) has a direct consequence on decreasing passengers average travel speed on the entire network. The main 
reason for limiting the number of stations for the underground network (excepting the aspects concerning the total investment costs) is the loss of efficacy induced by stops, stationing times and frequent start ups. Under these aspects, smooth disparities can be noticed between the present underground network and the one arising from the projected developments.

So, while the existing network has an average distance between the stations, $\overline{\mathrm{d}}=1.40 \mathrm{~km}$, with a dispersion $\sigma^{2}=0.19 \mathrm{~km}^{2}$, the projected network for 2030 will have $\overline{\mathrm{d}}_{1}=1.15 \mathrm{~km}$, with a dispersion $\sigma_{1}{ }^{2}=0.20 \mathrm{~km}^{2}$.

The average ubiquity of the network, $\overline{\mathrm{u}}$, in the above-mentioned hypothesis of a continuous space, can be defined as ratio between the number of stations, $\mathrm{n}$ and the total length of the network, $\sum \mathrm{L}_{\mathrm{r}}$ :

$$
\overline{\mathrm{u}}=\frac{\mathrm{n}}{\sum \mathrm{L}_{\mathrm{r}}}, \text { or } \overline{\mathrm{u}}=\frac{1}{\overline{\mathrm{d}}},
$$

expressed in number of stations for network's length unit (for example, km). The average value $\overline{\mathrm{u}}$ ubiquity dispersion is:

$$
\sigma_{\mathrm{u}}^{2}=\frac{1}{\mathrm{nd}^{2}} \sum\left(\frac{\overline{\mathrm{d}}}{\mathrm{d}_{\mathrm{i}}}-1\right)^{2} .
$$

For the existing network $\overline{\mathrm{u}}=0.71$ stations $/ \mathrm{km}$ and $\sigma_{\mathrm{u}}^{2}=0.10$ while for the developed network of $2030 \overline{\mathrm{u}}_{1}=0.87$ and $\sigma_{\mathrm{u} 1}^{2}=0.18$.

Comparing the values of ubiquities and their dispersions in the two hypotheses (existing network and the developed/extended one) the following conclusions can be drawn:

- the expected underground network development would bring an ubiquity increase and so a better served area,

- the significant values of ubiquity dispersion (and also for the distances between the stations) reflect the structural specificity of the area,

- $\quad$ the developed network is more adapted to the specificity of the served urban area $\left(\sigma_{u 1}^{2}>\sigma_{u}^{2}\right)$.

\subsection{Connexity}

Connexity is the network's property of assuring connexions among the considered points/areas.

It is obvious, by the representation of the planar graph associated with the network (only with the points/areas connected on the network), that connexity is assured. However, under these circumstances, infrastructure connexity must be extended to the service connexity for the main lines/routes of the underground network. From this point of view, if admitting that in all the junction points of the main underground lines there is the possibility of passing from one main line to another (even by passengers' travel from one platform to another, on the same level or from one station to another one situated on different levels), we can 
conclude that the network is connex (figure 2) both in the present and 2030 perspective.

\subsection{Connectivity}

The multiplicity of the connexions within a network represents connectivity. On a connective network, the possibility of choosing an itinerary between two nodes appears by comparing some alternatives.

As commonly the transport infrastructure networks do not have maximal connectivity (corresponding to a complete graph in which any node has direct connexion with all the other nodes) different indexes are used to reflect the network connectivity level. Among these, [6] for the actual case study we have used $\alpha$ and $\gamma$ indexes.

The $\alpha$ index is defined as ratio between the number of independent circuits of the graph associated to the network and the maximum possible number of independent circuits of the graph with the same number of nodes.

The number of independent circuits in a graph is given by the cyclomatic number of the graph:

where $\mathrm{A}$ is the number of links,

$$
\mu=\mathrm{A}-\mathrm{N}+\mathrm{G},
$$

$\mathrm{N}$ - number of nodes,

$\mathrm{G}$ - number of connected components,

and the maximum number of circuits of the planar graph is $2 \mathrm{~N}-3$, so:

$$
\alpha=\frac{\mu}{2 \mathrm{~N}-5}
$$

For the existing network $\alpha=0.015$ and for the extended one $\alpha_{1}=0.022$. The $\gamma$ index is determined as ration between the number of links of the graph associated to the network and the maximum number of links of the graph with the same number of nodes.

So, for the planar graph:

$$
\gamma=\frac{\mathrm{A}}{3(\mathrm{~N}-2)}
$$

The existing network has a $\gamma$ index of 0.166 while the extended one of 2030 has a $\gamma_{1}$ of 0.1 .

\subsection{Vulnerability}

Low values of connectivity both for the existing network and for the extended one can be notice (reflected by $\alpha$ and $\gamma$ indexes), meaning that the network (with almost a tree structure) is more vulnerable to losing the functionality of one or more links between the nodes than a complex network (following accidents or hyper congestion) $[1-4,8,12]$. For many of the links associated to the network's graph the lack of functionality might even mean losing connexity. These links (connecting marginal nodes and also the links 1-7, 7-15 and 10-11) are critical to network functioning. 
To the other links of the network a different vulnerability index can be credited in correlation with the consequences of prolonging the travel time necessary to assure all the connexions within the network while, successively, one link loses functionality.

The total length of the shortest paths between the nodes of the network (table 1) in case of all links functioning is $\sum \mathrm{L}=5978.9 \mathrm{~km}$ and in case of links successively losing functionality (figure 2) the sum of the shortest paths among the nodes of the network is $\sum \mathrm{L}_{\mathrm{d}}$ presented in table 2 .

The $\varepsilon$ ratio gives a measure of the vulnerability of the network's links in relation to the considered criterion. The links of the network are differentiated by $\varepsilon$ values that could become scale marks for a hierarchy of the links' importance in relation to network vulnerability. Bigger values of $\varepsilon$ signify links with high vulnerability (except the ones defined critical to the network as the loss of their functionality would bring network connexity loss too).

Table 2: $\quad$ The shortest path among the nodes of the underground network.

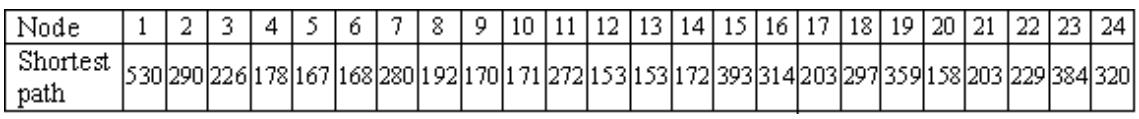

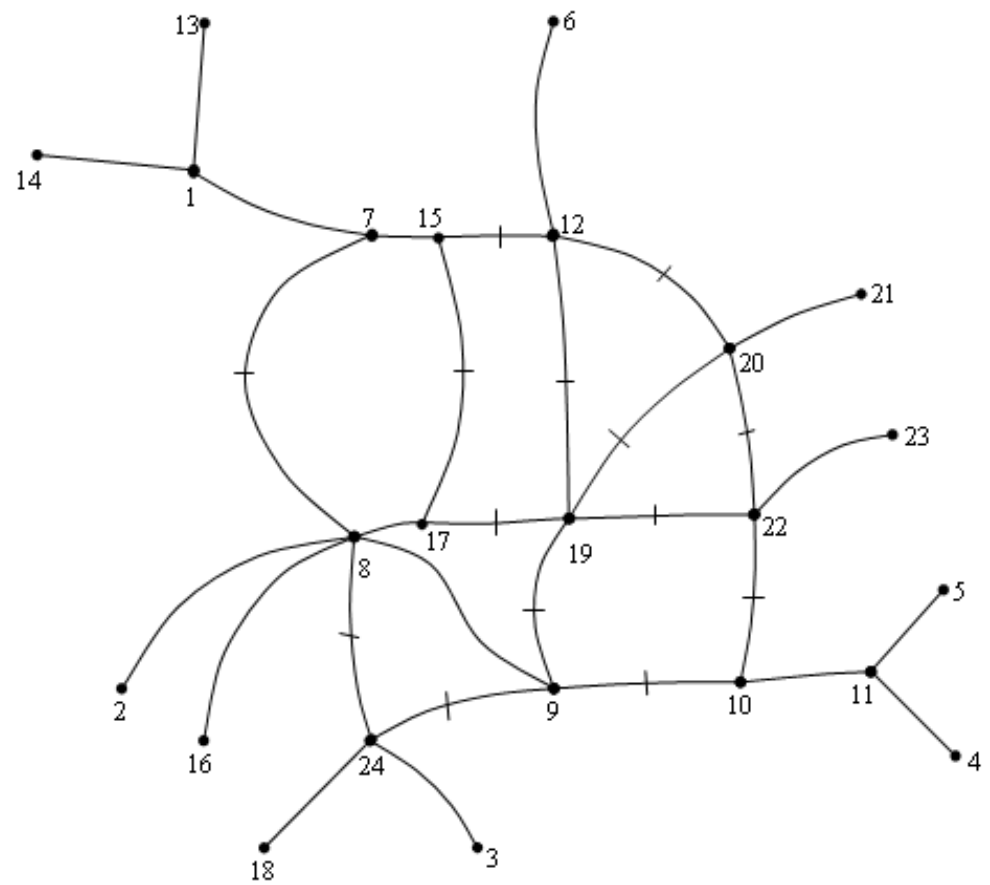

Figure 3: $\quad$ Simplified representation of the links of the underground network (the case of links losing functionality). 
Table 3: Sum of the shortest links among the nodes in the hypothesis of losing the functionality.

\begin{tabular}{|c|c|c|c|c|c|c|c|c|c|c|c|c|c|c|}
\hline Link that loses functionality & $7-8$ & $15-17$ & $17-24$ & 249 & $9-19$ & $19-17$ & $9-10$ & $19-22$ & $10-22$ & $20-22$ & $19-20$ & $19-12$ & $15-12$ & $12-20$ \\
\hline$\sum L_{d}$ & 5978.9 & 6152.28 & 5996.96 & 6084.38 & 6023.2 & 6165.72 & 6065.82 & 6018.5 & 6161.02 & 6154.22 & 6006.86 & 6029.74 & 6241.3 & 6086.5 \\
\hline$\varepsilon=\frac{\sum L_{d}}{\sum L}-1$ & 0 & 0.029 & 0.003 & 0.018 & 0.007 & 0.031 & 0.015 & 0.007 & 0.030 & 0.029 & 0.005 & 0.009 & 0.044 & 0.018 \\
\hline
\end{tabular}

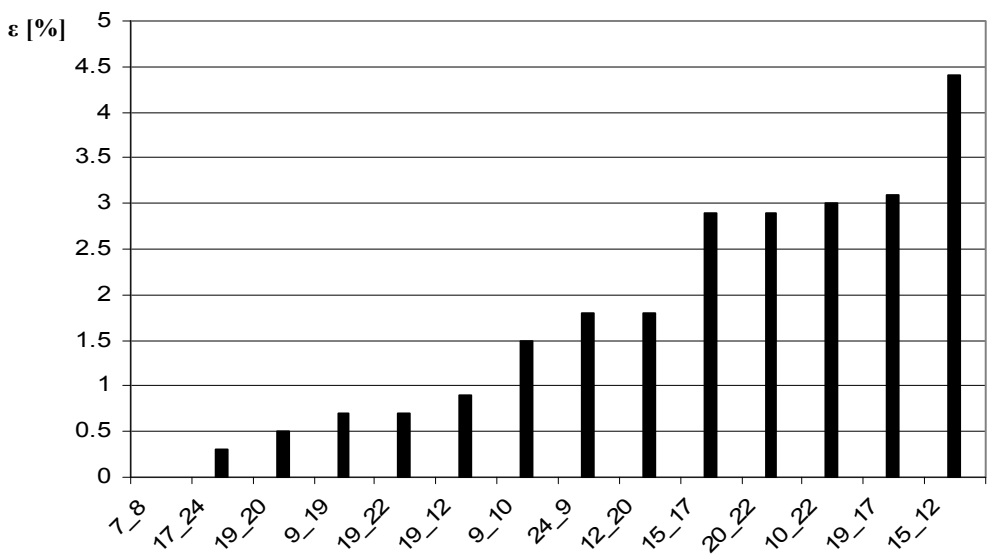

Link with functionality loss

Figure 4: Vulnerability of links with functionality loss.

The results present links 15-12, 19-17, 10-22, 20-22 and 15-17 as being the most vulnerable on the whole network.

\subsection{Homogeneity and isotropy}

These aspects are network properties that, in the strict way of definition [5], are idyllic, untouchable for any of the networks designed for material flow transfers.

In a wider approach, topological/geometrical/technical/functional characteristics of the network can be identified, that would allow the use of attributes like homogeneity/no homogeneity or isotropy/anisotropy for a transport network.

The previous references regarding ubiquity and vulnerability of the present and extended underground network revealed no homogeneity under the aspect of distances between stations and importance of the different links in assuring space-time correlations for the network. The links disparities from the vulnerability point of view can be interpreted as anisotropy, as they reveal the fact that not all the network's links are equivalent under the aspect of the relations assured among the network's elements. Actually, like properties of the networks, homogeneity and isotropy must be correlated to the relations assured among the elements of the network and not to irrelevant characteristics about 
those functioning links that the network assures for the use of the territory system. That is way the homogeneity of the underground network, under the aspect of the technical characteristics of infrastructure, transport means, technologies and even tariffs, is not presented as it is not relevant for network's homogeneity and isotropy from the functions to be assured point of view.

Relevant to the global characterization of the space-time correlations assured by the underground network we have considered to be the average transfer speed, $\bar{v}_{i}[7,9]$, from each i node to all the others, $j(j \neq i)$,

$$
\overline{v_{i}}=\frac{\sum_{j} d_{i j}}{\sum_{j} t_{i j}}
$$

where $d_{i j}$ is the distance from node $i$ to all the others, $j$,

$t_{i j}$ - travel time from node $i$ to node $j$ (including passengers' changing the line time in underground junction stations).

The izoaccessibility chart from figure 5 , drawn after grouping the $\bar{v}_{i}$ values of the extended network of 2030, shows no homogeneity and anisotropy of the network.

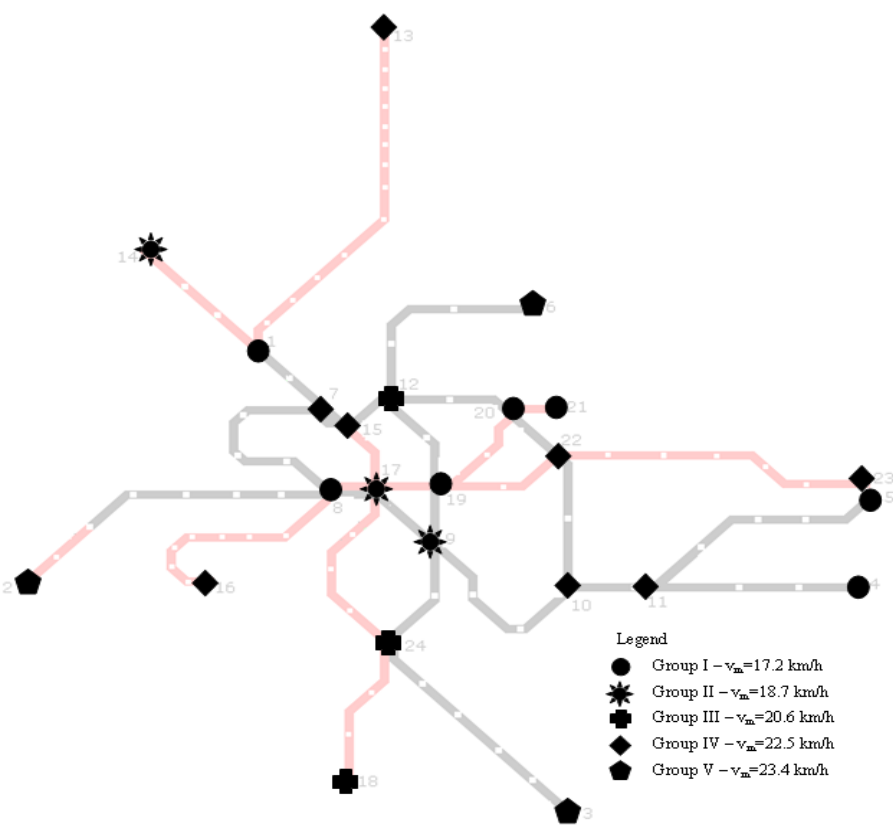

Figure 5: $\quad$ Izoaccessibility (for $\overline{\mathrm{v}}_{\mathrm{i}}$ values). 
Table 4: $\quad$ Shimbel accessibility and generalized accessibility indexes.

\begin{tabular}{|c|c|c|c|c|c|c|c|c|c|c|c|c|c|c|c|c|c|c|c|c|c|c|c|c|}
\hline Node & 1 & 2 & 3 & 4 & 5 & 6 & $?$ & 8 & 9 & 10 & 11 & \begin{tabular}{|l|}
12 \\
\end{tabular} & \begin{tabular}{|l|}
13 \\
\end{tabular} & \begin{tabular}{|l|}
14 \\
\end{tabular} & \begin{tabular}{|l|}
15 \\
\end{tabular} & \begin{tabular}{|l|}
16 \\
\end{tabular} & 17 & \begin{tabular}{|l|}
18 \\
\end{tabular} & 19 & 20 & \begin{tabular}{|l|}
21 \\
\end{tabular} & 22 & \begin{tabular}{l|l|}
23 \\
\end{tabular} & 24 \\
\hline$\left(\begin{array}{c}\mathrm{N} \\
(2010)\end{array}\right.$ & 35 & 35 & 31 & 37 & 37 & 31 & 25 & 25 & 21 & 21 & 27 & 21 & & & & & & & & & & & & \\
\hline $\begin{array}{l}\mathbb{N}_{0}, N_{0} \mathrm{Mar} \\
(2010) \\
\end{array}$ & 0.23 & 0.23 & 0.34 & 0.15 & 0.15 & 0.34 & 0.66 & 0.66 & 1.00 & 0.84 & 0.43 & 1.00 & & & & & & & & & & & & \\
\hline $\begin{array}{c}\mathbb{A}_{i} \\
(2030)\end{array}$ & 90 & 86 & 89 & 108 & 108 & 89 & 72 & 64 & 58 & 68 & 86 & $6 ?$ & 112 & 112 & 62 & 86 & 54 & 89 & 59 & 66 & 88 & 69 & 91 & $6 ?$ \\
\hline 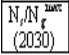 & 0.09 & 0.11 & 0.15 & 0.04 & 0.04 & 0.18 & 0.28 & 0.38 & 0.79 & 0.43 & 0.14 & 0.65 & 0.03 & 0.03 & 0.50 & 0.11 & 0.89 & $\mid 0.15$ & 1.00 & 0.68 & 0.19 & 0.63 & 0.17 & 0.55 \\
\hline
\end{tabular}

\subsection{Generalized accessibility}

As a measure of the relative importance of the nodes given by network's topology, the generalized accessibility [5, 7, 9], together with the other quantitative measures of network properties, completes the study's network characterization. Starting from the direct accessibility matrix, M, for the existing network, $\mathrm{M}^{2}, \mathrm{M}^{3}, \ldots, \mathrm{M}^{\mathrm{p}}$ and nodal vectors, direct accessibility vectors $\mathrm{N}_{1}$ and $\mathrm{N}_{\mathrm{i}}$, for $\mathrm{i}=\overline{1,5}$ were determined $\left(\mathrm{p}=5\right.$, network's diameter). Adding them $\left(\mathrm{N}_{\mathrm{i}}\right)$ gave us the generalized accessibility vector $\mathrm{N}_{\mathrm{g}}$ (table 4 ) and $\mathrm{n}_{\mathrm{g}}$ values, obtained by dividing $\mathrm{N}_{\mathrm{g}}$ to $\mathrm{N}_{\mathrm{g}}{ }^{\mathrm{max}}=420$, for the existing and the extended network.

Both for the existing and for the projected network one can see that the hierarchy of the nodes within the network is clearer as the nodal vector has a higher rank. The expected changes in network's configuration modify nodes' hierarchy. If in the present situation 9 and 12 nodes hold the best positions, in the future network they would be gained by 17 and 19 nodes.

\subsection{Shimbel accessibility}

Unlike generalized accessibility, the Shimbel accessibility takes into account only the direct links between the nodes $[5,7,9]$. Redundant links with repeated returns at origin and/or destination node are eliminated.

Shimbel accessibility vector (signifying the number of sequences - links involved in the connexion from that node to all the other nodes of the graph) emphasizes the dominant positions of the nodes 9,10 and 12 for the present situation and nodes 17 and 19 for the projected network (table 4).

\subsection{Nodal accessibility}

The generalized and Shimbel accessibility took into account only network's topology while the nodal accessibility $[5,7,9]$ differentiate network's nodes from the point of view of length, travel time and transfer cost for all the links between nodes' graph associated to the network (the links of the graph have geometrical and technical-functional characteristics associated, which integrates infrastructure aspects and technology performances of the network). Both for the existing and the projected network, the nodal accessibility, determined in relation to the transfer time between the nodes, led to the nodal accessibility vectors from table 5 . 
Table 5: $\quad$ Nodal accessibility vectors.

\begin{tabular}{|c|c|c|c|c|c|c|c|c|c|c|c|c|c|c|c|c|c|c|c|c|c|c|c|c|}
\hline Node & 1 & 2 & 3 & 4 & 5 & 6 & 7 & 8 & 9 & 10 & 11 & 12 & 13 & 14 & 15 & 16 & 17 & 18 & 19 & 20 & 21 & 22 & 23 & 24 \\
\hline $\begin{array}{l}\text { Travel time } \\
\text { (present) }\end{array}$ & 520 & 480 & 536 & 566 & 566 & 499 & 433 & 369 & 351 & 286 & 304 & 278 & & & & & & & & & & & & \\
\hline $\begin{array}{l}\text { Trawel time } \\
(2030)\end{array}$ & $m 2$ & 993 & 921 & 1284 & 1284 & 725 & 485 & 618 & 499 & 545 & 599 & 489 & 1428 & 922 & 440 & 841 & 502 & 83 & 525 & 571 & 637 & 456 & 720 & 597 \\
\hline
\end{tabular}

On the existing network nodes 12 (as a pole), 10 and 11 hold a dominant position, with close values and on the projected one nodes 15 (as a pole), 7, 9, 12 and 22 hold those positions. The upcoming underground network development outlines, by the dominant positions, the nodes situated in the central network area, in concordance to city's sights location (offices, high schools, universities, cultural sights, banks, etc.).

\section{Conclusions}

The structural changes of transport networks' infrastructure, following extension/reduction actions cannot be examined by the number of the links and/or nodes involved. They must be fit for the whole network needing strict evaluations of the attributes associated to their properties. The changes in any and all of the properties (connexity, connectivity, ubiquity, isotropy/ homogeneity, nodality) are the ones affecting network's functions of assuring territory need for mobility.

The comparative evaluation of Bucharest underground network for the present and future situation, using network properties, brought up complex aspects that are useful to the authorities in the field, in order to establish future directions of network extensions necessary to ameliorate its role and transport services within and to increase public urban transport attractiveness. We must notice the fact that the results of the study are not only based on the static examinations of the network for which the topological and geometrical characteristics are determinant. We also took into account the dynamic processes - consequences of service planning (lines/routes existing and projected, headways between the vehicles and passengers' transfer time in line junction points). That is why the study is useful to fundament tactic decisions of route choosing on a certain network configuration in relation to major passenger flows dynamics.

Without repeating the conclusions of the comparative calculations for the two networks in relation to every studied property and correlations within, we only emphasize the original manner of considering properties like ubiquity, vulnerability and isotropy.

\section{References}

[1] Bell, M.G.H., A game theory approach to measuring the performance reliability of transport networks, Transportation Research, vol. 34B, pp. 533-545, 2000. 
[2] Bell, M.G.H. and Y. Iida, Network reliability. Transportation network analysis, John Wiley \& Sons, pp. 179-192, 1997.

[3] Berdica, K., An introduction to road vulnerability: what has been done, is done and should be done, Transport policy, 9(2), pp. 117-127, 2002.

[4] Chen, A., H. Yang, H.K. Lo, W. H. Tang, A capacity related reliability for transportation networks, Journal of Advanced Transportation, vol. 33(2), pp. 183-200, 1999.

[5] Dupuy, G., Systemes, reseaux et territories: principe de reseautique territorial, Presses de l'ENCP, 1985.

[6] Kansky, Structure of Transportation Networks: Relationships between Network Geometry and Regional Characteristics, Chicago University Press, 1963.

[7] Mackiewicz, A., Ratajczak, W., Towards a new definition of topological accesibility, Transportation Research, 30B/ 1, 1996, pp. 47-79.

[8] Raicu, S. et al. Researches about transport network reliability, PNCDI Grant, Education and Research Ministry, 2004.

[9] Raicu, Ş., Sisteme de transport, Ed. AGIR, Bucureşti, 2007.

[10] Raicu, S. et al. Solutions for the attractivity increasing of the urban public transportation. Case study: Bucharest city and its metropolitan area. (in Romanian: Soluţii pentru creşterea atractivităţii transportului public urban. Studiu de caz pentru Bucureşti şi aria metropolitană) research project, UPB, 2006.

[11] Raicu, Ş. et al. About the high capacity public transport networks territory functions, Urban Transport XV - Urban Transport and the Environment, Editor C.A. Brebbia, WIT Press, pp. 41-51, 2009.

[12] Taylor, M.A.P., D’Este, G.M., Critical infrastructure and transport network vulnerability, The Second International Symposium on Transportation Network Reliability (INSTR), New Zealand, pp. 96-103, 2004. 